\title{
Fragmentation et engagement dans Eles eram muitos cavalos, de Luiz Ruffato
}

\author{
Fragmentação e engajamento em Eles eram muitos cavalos, de Luiz Ruffato
}

Fragmentation and engagement in Eles eram muitos cavalos, of Luiz Ruffato

\author{
Ilana Heineberg \\ Université Bordeaux Montaigne - Bordeaux - France
}

$\diamond$

Résumé: Cet article - qui introduit l'écrivain Luiz Ruffato et son roman Eles eram muitos cavalos (Tant et tant de chevaux) à un public francophone - part du principe que l'auteur emploie des stratégies littéraires postmodernistes sans renoncer à une prise de position par rapport aux problèmes sociaux du Brésil. Ruffato serait, donc, exemplaire d'un incontestable engagement postmoderne, contredisant des théoriciens marxistes, comme Frederic Jameson et Terry Eagleton, qui conçoient la littérature postmoderniste comme un vide idéologique. Nous commencerons par analyser les multiples formes de fragmentation du texte qui constituent, pour Ruffato, une stratégie de dénonciation sociale. Puis, nous verrons comment les différentes perspectives narratives adoptées dans certains fragments du roman permettent de situer la figure de l'auteur par rapport à ses personnages et, ainsi, énoncer l'éthique ruffatienne.

Mots-clés: Luiz Ruffato; Eles eram muitos cavalos; Fragmentation; Engagement; Postmodernisme

Resumo: Esse artigo - que introduz o escritor Luiz Ruffato e seu romance Eles eram muitos cavalos a um público francófono - parte do princípio que o autor emprega estratégias literárias pós-modernistas sem abrir mão de uma literatura empenhada com os problemas sociais do Brasil. Ruffato seria, portanto, exemplar de um engajamento autenticamente pós-moderno, contrariando teóricos marxistas, como Frederic Jameson e Terry Eagleton, que veem no pós-modernismo o fim de todas ideologias. Começaremos por analisar as múltiplas formas de fragmentação do texto que constituem, para Ruffato, uma estratégia de denúncia social. A seguir, veremos como as diferentes perspectivas narrativas adotadas em certos fragmentos do romance permitem situar a figura do autor em relação a seus personagens e, desse modo, enunciar a ética ruffatiana.

Palavras-chave: Luiz Ruffato; Eles eram muitos cavalos; Fragmentação; Engajamento; Pósmodernismo

\begin{abstract}
This study - which introduces the writer Luiz Ruffato and his novel Eles eram muitos cavalos to a francophone public - assumes that this Brazilian writer employs postmodernists literary strategies without giving up a literature committed to the social problems of Brazil. Ruffato would be, therefore, a genuine example of post-modern social engagement, and, in this way, contradicts Marxist theorists, such as Frederic Jameson and Terry Eagleton, who see in the post-modernism the end of all ideologies. The paper begins examining the multiple forms of fragmentation of the text, what Ruffato believes to be a strategy of social complaint. Next, its looked at how the different narrative perspectives are used in certain fragments of the novel and how they allow one to situate the figure of the author in relation to their characters, in a way that Ruffato`s ethics it is enunciated.
\end{abstract}

Keywords: Luiz Ruffato; Eles eram muitos cavalos; Fragmentation; Engagement; Postmodernism 
Le nom de Luiz Ruffato est l'un des plus connus de la littérature brésilienne actuelle. Son livre Eles eram muitos cavalos $^{1}$ (Tant et tant des chevaux), de 2001, connaît un véritable engouement de la part du public et de la critique, comme l'attestent plusieurs prix, traductions et travaux universitaires ${ }^{2}$. Ruffato constitue le parfait exemple de la question que nous nous posons $\mathrm{ici}^{3}$, celle de la possibilité d'avoir et de défendre, à travers la littérature, des engagements, notamment sociaux, dans une littérature postmoderniste. Si la lecture de certains théoriciens du postmodernisme ${ }^{4}$ pourrait nous faire conclure que l'engagement n'a aucune place possible dans un roman postmoderniste, le cas de Ruffato nous permet de résoudre ce paradoxe apparent et de vérifier les contours adoptés par l'engagement social dans la littérature brésilienne contemporaine.

La violence pratiquée ou subie par des personnages souvent marginaux de la société inégalitaire brésilienne constitue la thématique dominante de Eles eram muitos cavalos, à l'instar de celle d'autres écrivains, réalisateurs et scénaristes de cinéma et de séries télévisées qui ont une portée internationale ${ }^{5}$. Si Ruffato puise dans l'inévitable terreau de la violence ${ }^{6}$, il s'éloigne du courant dit "brutaliste" par une critique sociale explicite qui ne laisse aucune place à la banalisation du phénomène. Ruffato s'inscrit par là dans la tradition réaliste brésilienne ${ }^{8}$,

\footnotetext{
Toutes les citations du corpus seront données entre parenthèses avec l'abréviation EEMC et le numéro de la page. L'édition consultée: RUFFATO, Luiz. Eles eram muitos cavalos. 7a. ed. rev. Rio de Janeiro: Record, 2007. Édition française consultée: RUFFATO, Luiz. Tant et tant de chevaux. Traduit du brésilien par Jacques Thiériot. Paris: Métailié, 2005.

2 Eles eram muitos cavalos a reçu le prix meilleur roman de l'Associação Paulista de Críticos de Artes et le prix Machado de Assis, dans la catégorie récit, de la Fundação Biblioteca Nacional. Il a été traduit en italien (2003), français (2005), espagnol (2010), allemand (2012) et finlandais (2014) et a été publié au Portugal en 2006. Des dizaines de thèses, de mémoires de master et d'articles universitaires ont été écrits sur ce livre, dont un recueil d'essais entièrement consacré à Eles eram muitos cavalos: HARISSON, Marguerite Itamar (Org.). Uma Cidade em camadas: ensaios sobre Eles eram muitos cavalos de Luiz Ruffato. Vinhedo: Ed. Horizonte, 2007.

3 Les propos développés dans ce texte ont fait initialement l'objet d'une communication présentée lors du colloque Savoir et pouvoir à l'ère de la "modernité liquide": transformations et enjeux des formes de l'engagement littéraire de la fin $d u X X^{e}$ au XXI siècle, organisé par Martine Bovo Romeuf et Ana Maria Binet et réalisé le 9 et 10 juin 2011 à l'Université Bordeaux Montaigne.

4 C'est la critique que des théoriciens marxistes comme Frederic Jameson (2004) et Terry Eagleton (1998) font à certains écrivains et théoriciens postmodernistes.

5 Les exemples les plus connus en France sont Cidade de Deus (2002), de Paulo Lins, adapté au grand écran par Fernando Meirelles, puis repris dans la série Cidade dos homens (2007). Le roman de Marçal Aquino, $O$ Invasor, fait le chemin inverse; il est publié après la sortie du long métrage homonyme, qu'il a écrit pour être réalisé par Beto Brant en 2001. Enfin, le film Tropa de elite, de José Padilha (2007) est récompensé par l'Ours d'or à Berlin et son deuxième volet attire plus de 10 millions de spectateurs dans les salles de cinéma en 2010.

6 Pour João Cézar Castro Rocha, c'est le remplacement partiel de la "dialectique du malandresque" (CANDIDO, 1970 et CANDIDO,
}

tout en la déconstruisant à travers des procédés formels qui l'aident à problématiser cette réalité.

L'ensemble de l'œuvre que Ruffato est en train de construire atteste de ses engagements littéraires et socio-politiques. Depuis 2005, il publie la série Inferno provisório", qu'il définit comme un "roman non-bourgeois"10. On y trouve l'histoire du prolétariat provincial brésilien à partir de sa constitution dans les années 1950 jusqu'à la migration de la campagne vers des noyaux urbains plus importants dès les années 1970. Consacré justement aux conséquences actuelles de ce processus de "modernisation", Ruffato a déjà évoqué l'hypothèse (BUARQUE DE HOLLANDA) que Eles eram muitos cavalos, situé en l'an 2000, à São Paulo, mégalopole de 20 millions d'habitants, devienne un volume de la série.

Cependant, il ne faut pas s'attendre à trouver chez Ruffato une saga larmoyante du prolétariat brésilien ayant pour toile de fond l'histoire du pays. C'est là qu'il faut évoquer, à mon sens, le postmodernisme et quelquesunes de ses stratégies littéraires. Loin de faire d'Inferno provisório un roman fleuve ou un roman historique, Ruffato dialogue avec l'histoire et son écriture en proposant une autre perspective, celle des seconds rôles anonymes, des "ex-centriques", pour utiliser le terme de Linda Hutcheon nommant ce ou ceux qui ne trouvent pas

1995) par la "dialectique de la marginalité" qui expliquerait que le dénominateur commun d'un grand nombre de productions culturelles récentes brésiliennes soit une image d'un pays défini par la violence. Selon 1'essayiste, "La 'dialectique de la marginalité' suppose une nouvelle forme de relation entre les classes sociales. Il ne s'agit plus de concilier des différences, mais de les souligner, en récusant l'improbable promesse d'un compromis entre le cercle restreint des maîtres du pouvoir et l'univers croissant des exclus"' (ROCHA, 2005, p. 70-71).

7 L'adjectif "brutaliste" employé par le critique Alfredo Bosi renvoie aussi à une écriture "rapide, compulsive parfois obscène" caractéristique de ce monde violent (BOSI, 2002:18)

8 Selon Karl Erik Schøllhammer, le nouveau réalisme conjugue l'ambition d'être à la fois "référentiel" - sans être forcément représentatif et "engagé" - sans s'inscrire nécessairement dans un programme politique ou sans vouloir transmettre de façon coercitive des contenus idéologiques. L'essayiste cite un entretien de Luiz Ruffato au supplément Ilustrada du journal Folha de São Paulo (71/03/2005), où celui-ci se présente comme héritier du courant réaliste et, en même temps, affirme s'éloigner à la fois du néonaturalisme et de la littérature égotique intimiste contemporaine: "Je suis, d'une certaine manière, dans le contre-courant de la littérature contemporaine brésilienne. Celle-ci révèle un penchant soit pour le néonaturalisme soit pour une littérature que j'appelle égotique, trop centrée sur le moi. J'essaie de prendre un autre chemin, celui de la littérature réaliste, qui, à mon avis, n'est ni optimiste, ni pessimiste. Elle établit une réflexion sur le réel à partir du réel" (apud SCHØLLHAMMER, 2009, p. 54-55). Traduit par nos soins.

9 Il s'agit de Mamma son tanto felice (2005), O mundo inimigo (2005), Vista parcial da noite (2006), O livro das impossibilidades (2008) et Domingos sem Deus (2011). En France, les éditions Métailié ont publié jusqu'à présent les deux premiers tomes de la série: Des gens heureux (2007) et Le monde ennemi (2010).

10 Au-delà de faire référence à la thématique de la série, le terme "roman non-bourgeois" renvoie également à l'aspect formel fragmentaire du texte, qui s'oppose à la forme du roman classique. (BUARQUE DE HOLLANDA, 2016). Voir également note 13. 
leur place au centre ${ }^{11}$. Outre cette révision de l'histoire à travers les microhistoires des exclus des livres et des manuels scolaires, Ruffato propose à ses lecteurs une écriture fragmentaire, propre aussi à la postmodernité.

Je vous propose donc d'analyser l'engagement postmoderne de Ruffato dans Eles eram muitos cavalos, en dehors de toute considération sur une éventuelle tentative de l'auteur de répondre à une demande de l'industrie culturelle (cf. CORPAS, 2009). Je m'intéresserai, d'abord, à l'aspect fragmenté de Eles eram muitos cavalos, ce qui me permettra, outre de vous donner un aperçu du roman, d'expliciter la principale stratégie postmoderniste qui appuie cette dénonciation. Puis, nous verrons comment l'adoption d'une perspective narrative à la fois proche et distante des personnages permet à Ruffato de faire part de son éthique.

\section{Éclatement du texte}

Eles eram muitos cavalos est un roman qui n'en est pas un. Il s'agit d'une juxtaposition de 69 fragments numérotés, désignés par un titre et portant sur les situations les plus diverses vécues par des habitants de la ville de São Paulo dans la journée du 9 mai 2000. Les Paulistanos de Ruffato sont, majoritairement, anonymes ${ }^{12}$ et appartiennent aux milieux défavorisés ou à la classe moyenne basse, étant confrontés à des situations de précarité. Mais on reconnaît également São Paulo à sa minorité très aisée: des hommes politiques corrompus, un chef d'entreprise se déplaçant en hélicoptère, un père qui, conduit par un chauffeur, va chercher son fils à l'école pour déjeuner au Mc Donald's et se révèle être un trafiquant d'armes, une jeune chanteuse de rock, des habitants d'ensembles sécurisés.

Chaque fragment propose une solution narrative différente et unique pour raconter son intrigue. Une telle inventivité formelle nous signale qu'un récit traditionnel et homogène ne pourrait pas rendre compte ni des différents langages parlés à São Paulo, ni des différents mondes qui habitent cette ville ${ }^{13}$. Ainsi, Ruffato alterne le recours au discours direct, aux flux de conscience et aux discours indirects libres, avec une préférence marquée pour ces

\footnotetext{
11 La théoricienne canadienne Linda Hutcheon emploie le terme "excentrique" pour signifier un décentrement de classe, de race, d'orientation sexuelle ou d'ethnie propre au postmodernisme. Selon elle, dans un paradoxe typiquement postmoderne, l'ex-centrique est inévitablement identifié au centre auquel il aspire, mais qui lui est nié (Cf. HUTCHEON, 1991, p.88). Dans cet article, j'emploie cette notion pour signifier, évidemment, l'exclusion sociale.

12 Sur l'anonymat dans Eles eram muitos cavalos, voir l'essai: WALTY, Ivete Lara Camargos. Anonimato e resistência em Eles eram muitos cavalos. In: HARRISON, Marguerite Itamar (Org.), Uma Cidade em camadas: ensaios sobre Eles eram muitos cavalos de Luiz Ruffato. Vinhedo: Ed. Horizonte, 2007.

13 Dans l'entretien du 14/03/2005 au supplément Ilustrada du quotidien Folha de São Paulo, Ruffato parle de l'importance du rapport entre
}

derniers. En effet, le discours indirect libre lui permet de donner voix à des personnages exclus dont le langage, sans l'aide du narrateur, n'est pas capable d'assurer une communication complexe. À travers la polyphonie, Ruffato oblige son lecteur à déchiffrer ces différents dialectes et accents, à donner une place à l'autre, à celui qui est différent de soi. Souvent, les voix sont "enrichies" par des caractères gras ou italiques, s'approchant par là du langage théâtral.

Par ailleurs, à travers plusieurs recours, la prose devient souvent poétique dans Eles eram muitos cavalos. Le découpage d'un fragment en vers en est un exemple. Ruffato emploie cet effet aussi bien dans des fragments entiers que dans des extraits pour mettre en avant une information ou pour susciter une émotion particulière. Pour les fragments entièrement en vers, cela peut concerner des discours qui, à l'origine, n'ont rien de littéraires, comme le fragment "7. 66", la prévision numérologique de la journée, ou le "36. Leia o Salmo 38", une recette d'un rituel superstitieux pour exaucer un vœu (qu'on appelle simpatia au Brésil) ; mais le choix de versifier un fragment entier peut concerner aussi des textes déjà chargés d'émotion, comme dans "34. Aquela mulher", où le protagoniste, après la mystérieuse disparition de sa fille, erre à travers les "ruesavenues du morumbi". Quant à l'utilisation occasionnelle du vers dans un fragment, elle peut, par exemple, contribuer à une impression de chute libre. Tel est le cas de la fin du fragment "10. O que quer uma mulher":

?quem é esse homem, meu deus,
$\quad$ cara gorda ponte-móvel
barriga-de-barril roupas desleixadas sem amigos
que gasta as tardes de domingo vendo futebol
$\quad$ na televisão
latinhas de cerveja e tira-gostos espetados no palito
e que dorme em sua cama
e que é o pai de seus filhos
e que
meu deus
já não conhece
quem é esse homem quem?
(EEMC, p. 30).

\footnotetext{
le fond et la forme dans son œuvre: "La forme classique du roman a été façonnée pour régler des questions qui se posaient au début de la révolution industrielle. Ensuite, elle a dû s'adapter selon l'époque, en arrivant jusqu'à James Joyce. L'instrument roman, avec début-milieufin, ne fait plus sens devant la quantité d'informations que nous avons à notre disposition aujourd'hui; il est devenu obsolète. Mon choix du fragmentaire a été véritablement une provocation. Quand j'ai publié Eles eram muitos cavalos, beaucoup de critiques ont pincé le nez en disant 'mais ceci n'est pas un roman'. Je pense aussi que ça ne l'est pas. Mais c'est quoi alors? Ce n'est pas un recueil de nouvelles non plus. Je veux mettre à l'épreuve toutes ces structures. Je ne veux pas faire seulement une réflexion sur la réalité politique, mais aussi poser des questions par le biais du contenu et de la forme" (apud SCHØLLHAMMER, 2009, p. :55). Traduit par nos soins.
} 
L'histoire de Crânio, jeune de la favela "qui ne fume pas ne sniffe pas" et qui "passe toute la journée à lire", est racontée par son frère dans le fragment " 47 . O "Crânio"" à travers des vers assez longs et saccadés, évoquant la musicalité du rap ou du funk.

Les figures de continuité phoniques contribuent également à rendre poétique la prose. Si l'allitération et l'anaphore sont souvent perdues ou déplacées dans la traduction française, en portugais, nous les trouvons dans le fragment " 35 . Tudo acaba" (les caractères gras et soulignés sont de notre fait):

o sujeito no farol se assusta
atira
e o cara sangrando sobre o volante o carro ligado
o povo puto atrás dele
ele
atrapalhando o trânsito
(EEMC, p. 77$)$

Dans la traduction française du fragment "45. Vista parcial da cidade", les souvenirs de la campagne de la passagère âgée d'un bus contiennent des répétitions sonores qui contribuent à rendre la mémoire plus vive, tout en se mélangeant avec les bruits du bus (les caractères gras et soulignés sont de notre fait): "la corde chante sur la poulie le seau apporte un peu d'eau saumâtre le silence des vaches qui mugissent la sécheresse roussie entre les orteils" (RUFFATO, 2005, p.98).

Finalement, l'usage de parallélismes contribue à l'aspect poétique du texte. La reprise d'un même patron syntaxique a une valeur à la fois démonstrative et émotive, appuyant une rhétorique de l'empilement, qui peut renvoyer, dans le fragment "62. Da última vez", à une répétition exaspérante qui conduira à la séparation du couple:

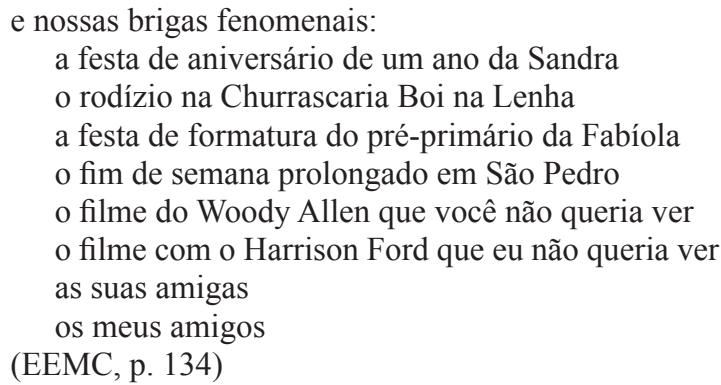

Outre l'hybridation des genres littéraires, Ruffato insère ou reproduit des textes appartenant à d'autres genres discursifs: chaîne de prière, météorologie, petites annonces d'emploi, sexe ou amitiés, carte d'un restaurant chic, horoscope, lettre, etc. Lorsque ces textes éphémères, voués à la déchetterie et à l'oubli des citoyens, sont recyclés et juxtaposés à des textes littéraires, ils gagnent un tout autre statut: celui de ce que j'appellerai ici "résidus textuels de la ville". Par ailleurs, ces résidus contaminent les situations d'êtres souvent anonymes auxquels ils apparaissent juxtaposés, pointant ce qui, dans la vie de ces hommes, relève de l'éphémère, du jetable, voire du déchet, comme nous le verrons par la suite.

\section{Fragments, résidus et dénonciation}

Par ses formes inventives, Ruffato s'inscrit, certes, dans la continuité des expériences modernistes ${ }^{14}$, mais cet usage du collage, du fragmentaire et de l'hybridation des genres littéraires et discursifs est surtout un moyen de représenter la société paulistana postmoderne. Cela, tout en mettant en évidence la crise de la représentation pour dire le rythme hallucinant, le mode de communication des nouvelles technologies, la précarité, l'insécurité, l'individualisme, la solitude et la consommation comme moyen d'appartenance sociale. L'utopie et l'euphorie que le phénomène urbain inspirait chez les modernistes sont remplacées, dans la postmodernité, par le désenchantement. La fragmentation ne semble plus une provocation, mais une constatation de l'état et de la place du fragment qui est de plus en plus dissocié d'un tout.

L'étymologie du mot fragment (du latin fragmentum du radical du verbe frangere, "briser, rompre") selon la définition donnée par le dictionnaire ("morceau d'une chose matérielle qui a été cassée ou brisée", selon $L e$ Robert) met en évidence le lien entre le fragment et la dissolution, la décomposition. Chez Ruffato, comme dans l'esthétique contemporaine en général, l'omniprésence du fragment métaphorise évidemment une société en ruine.

Issu d'une déchirure, le fragment séparé du tout devient alors résidu. Dans Eles eram muitos cavalos, cette association est également exploitée. Comme Zygmunt Bauman (2009) l'a exhaustivement démontré dans Vies perdues: la modernité et ses exclus, dans une société qui produit, consomme et jette de plus en plus, l'homme devient aussi déchet, rebut. Ainsi, dans le fragment "23. Chegasse o cliente", les cadavres de deux ouvriers tombés des échafaudages sont relégués au statut d'ordures. Les corps sont décrits de façon déshumanisée (les caractères gras sont de notre fait):

o rosto de um esborrachado contra a guia a perna sobre as costas um malabarismo agora inútil pelicano desengonçado outro saco-de-estopa onde apressado alguém em evidente flagrante tivesse enfiado um monte de ossos ou ainda um relógio-despertador desmontado uma engrenagenzinha uma mola um (EEMC, p. 53)

\footnotetext{
${ }^{14}$ Karl Erik Scholhammer défend les racines modernistes des expériences de Luiz Ruffato dans: Fragmentos do real e o real dos fragmentos. In: HARRISON, Marguerite Itamar (Org.). Uma cidade em camadas: ensaios sobre Eles eram muitos cavalos de Luiz Ruffato. Vinhedo: Horizonte, 2007
} 
Leur présence devient gênante, imposant au restaurant qui se trouve sur le trottoir un ménage urgent, comme le souligne l'ouverture du texte: "com um balde amarelo de plástico cheio de água azulada de sabão em pó e uma vassoura de pelo sintético amarelo os dois faxineiros rapidamente lavaram o cimento" (EEMC, p. 52-53). De même, ceux qui survivent de ce que la société rejette deviennent pour celle-ci des rebuts humains; les clochards, dans une grande ville, n'inspirent que le dégoût ou l'indifférence. Ils sont les personnages des fragments "11. Chacina no 41", "34. Aquela mulher" et "66. Rua", qui redonnent une dimension humaine à ces hommes et femmes.

Les excrétions et leurs puanteurs apparaissent dans plusieurs fragments. La mère et ses nombreux enfants misérables du fragment "9. Ratos" partagent le lit avec des rats. Les rongeurs, les premiers à être vus par le narrateur, s'alimentent aussi bien d'excréments humains ("[um rato] experimenta mastigar um pedaço de pano emplastrado de cocô mole, ainda fresco", [EEMC, p. 22]) que de "quelque chose de doux et chaud" qui se révèle être la "chair tendre" d'un bébé. Dans l'autocar GaranhunsSão Paulo ("6. Mãe"), "l'air pestilentiel des fenêtres fermées", "les vitres qui suintent", "la puanteur du réduit au bout du couloir" ne sont que le prolongement de la passagère dont "la vessie [est] ballonnée" et "les intestins prêts à se relâcher". Dans l'école primaire dégradée du fragment "13. Natureza-morta", les malfaiteurs laissent "une ardoise par terre couverte de vomi", "des pinceaux imbibés d'excréments qui ont gribouillé des abstractions sur les murs blancs", "une bouteille de Coca-cola pleine de pisse" en guise de signature. L'Indien parachuté dans un quartier modeste ("14. Um índio") arrive à avoir un peu d'alcool et des restes de nourriture, mais on lui présente le prix: "Toma, vai lavar o banheiro primeiro [...] Depois, o piso... Essa imundicie (EEMC, p. 34-35)". De même, la présence d'ordures dans les rues décrite de manière aussi banale que récurrente atteste de la valeur, ou plutôt du manque de valeur, attribuée à l'espace public. Dans le fragment "16. Assim:", l'homme d'affaires qui se déplace en hélicoptère évoque la dégradation du centreville: "irreconhecivel o centro da cidade hordas de camelôs batedores de carteiras homens-sanduiche cheiro de urina cheiro de óleo saturado cheiro de [...] (minha mãe punha luvas, chapéu, salto-alto para passear no viaduto do chá" (EEMC, p. 39).

Le lexique du déchet, de la pourriture n'épargne pas les milieux sociaux les plus aisés. Dans le fragment "25. Pelo telefone", une femme laisse huit messages sur le répondeur téléphonique de la jeune maîtresse de son mari. Elle finit par prévenir celle-ci, avec une richesse de détails, du mauvais usage que l'homme fait des toilettes, laissant systématiquement des traces de ses excréments.
La fragmentation est présente également à l'intérieur de chaque extrait. Cela arrive, premièrement, lorsque le narrateur de Ruffato semble être entré en retard en scène et que le lecteur doit reconstituer l'histoire, comme une conversation intéressante que l'on surprend in medias res. Le fragment "51. Política", sur les débauches d'un député, en est un bon exemple. Le texte s'ouvre en minuscule, ce qui donne au lecteur l'impression d'avoir manqué la partie la plus croustillante des révélations: "não posso declinar o nome dele, entende?, ele é muito conhecido, vira e mexe tem retrato dele no jornal, a cara dele aparece na televisão" (EEMC, p. 113).

La fragmentation interne se fait sentir également par des fins intempestives où non seulement l'intrigue est interrompue, mais aussi la phrase elle-même. Les exemples sont très nombreux. Dans le fragment "15. Fran", une comédienne décadente passe la journée à boire et à attendre que le téléphone sonne pour une offre de travail, ce qui, bien évidemment, ne viendra pas. À la fin, la phrase coupée de la secrétaire ne fait que confirmer l'échec de Fran: "Ah, Augusto, velho Augusto, bom Augusto, no celular sempre a secretária-eletrônica, Deixe seu recado após, no escritório a Miriam Deixa comigo, meu bem, assim que puder ele retorna sua ligação, ele já sabe do que se trata, pode ficar" (EEMC, p. 39). Fran auraitelle raccroché ? Ou aurait-elle tout simplement éloigné le combiné de l'oreille, connaissant par cœur cette réplique? En tout cas, le lecteur, avec Fran, peut facilement compléter la réponse, ce qui révèle le caractère faux de celle-ci.

Que le texte apparaisse coupé au début ou à la fin, ce recours textuel expose le constat postmoderne de l'impossibilité de penser les situations de façon évolutive, avec un début, un développement et une fin. Celles-ci sont plutôt en devenir et il serait aussi absurde que rassurant de vouloir les apprivoiser dans un ensemble cohérent. Montrant une São Paulo où l'on ne peut pas se sentir rassuré, l'auteur prend le parti de renforcer le sens de cette fragmentation. En outre, cette apparence de hasard que Ruffato prête à certains fragments, soit parce qu'on arrive un peu trop tard, soit parce qu'on part trop tôt du déroulement d'une scène, rappelle finalement qu'un récit commence et finit là où l'auteur le décide. Mettre en avant l'arbitraire de l'écriture fictionnelle, tout en feignant le hasard, revient, finalement, à mettre au jour les échafaudages du texte.

La fragmentation interne se fait sentir en dernière instance par la discontinuité des discours des personnages ou des narrateurs, souvent interrompus par d'autres, ou submergés par les bruits de la ville ou encore tout simplement tus par manque d'écoute. Les phrases sans connecteurs, dont les mots apparaissent juxtaposés, octroient au lecteur un rôle actif dans le remplissage des lacunes. 


\section{Les parties et le tout}

Présent à tous les niveaux du texte ruffatien, le fragment finit ainsi par devenir un fil conducteur. Ce texte fragmenté a encore d'autres connecteurs.

Le choix des épigraphes en est un. La première, qui explique également le titre, est issue de Romanceiro da Inconfidência ${ }^{15}$ (1953), de Cecília Meirelles: "Eles eram muitos cavalos,/ mas ninguém mais sabe os seus nomes,/ sua pelagem, sua origem..." évoque les animaux anonymes (Walty, 2007, p.56) qui ont aidé à faire la première rébellion coloniale contre la métropole portugaise $^{16}$ (1789). Nous avons donc ici, les thèmes de l'anonymat, de l'oubli et des figurants de l'histoire qui sont ceux de plusieurs fragments du roman de Ruffato. La deuxième épigraphe est puisée dans le psaume 82 de la Bible: "Jusqu'à quand jugerez-vous injustement en soutenant la cause des impies ?" Si la deuxième personne du pluriel est employée par Dieu dans le texte sacré pour s'adresser à des juges, lorsque la citation est reprise par Ruffato, le pronom peut renvoyer au lecteur lui-même. Devenu juge à son tour, c'est la passivité. et l'injustice de ce dernier qui sont questionnées. Il y a donc une interrogation sur l'engagement du lecteur.

Les trois premiers textes-fragments du livre indiquent aussi d'autres points de convergence. Le premier, intitulé "En-tête", permet de situer dans un même espace et dans un même temps tous les textes: "São Paulo, 9 de maio de 2000. Terça-feira", un jour quelconque. Cela pourrait être un radioréveil qui semble ouvrir une longue journée qui sera mise en scène tout au long du livre, du petit matin de ce mardi jusqu'à la nuit du mercredi. Le fragment "2. Le tempo", n'est que la prévision météorologique de la journée, poussant également à l'extrême le langage référentiel omniprésent dans le roman. Enfin, ce n'est pas par hasard si le fragment "3. Hagiologia" évoque sainte Catherine de Boulogne, une protectrice des pauvres, eux-mêmes personnages de la plupart des fragments. C'est seulement à partir du quatrième fragment que commencent les textes narratifs.

Deux événements factuels qui apparaissent de façon disséminée dans plusieurs textes fonctionnent également comme des fils conducteurs entre les divers fragments: la fête des mères et le match de Corinthians, l'équipe paulistana préférée des couches populaires. Ayant lieu au

\footnotetext{
${ }^{15}$ Les vers utilisés par Ruffato dans le titre et dans l'épigraphe appartient au "Romance LXXXIV ou Dos cavalos da Inconfidência" (MEIRELES, 2001, p.962)

16 Comme le souligne Walty (2007,p. 57), par la reprise du genre médiéval ibérique romanceiro (formé par plusieurs romances, chant proche de la ballade), Cecília Meireles faisait elle aussi le choix du fragmentaire et de l'hybride. En outre, l'histoire de l'Inconfidência Mineira y est subvertie, car Cecília Meireles donne une place d'honneur aux légendes, aux croyances populaires et aux personnages marginaux.
}

Brésil, chaque deuxième dimanche du mois de mai, la fête des mères, en 2000, est tombée précisément le 14 mai, le dimanche qui suit le mardi 9. Dans quelques extraits, les personnages y font référence comme s'il s'agissait de l'accomplissement d'une activité quotidienne, qui peut être banale: "ih tenho que comprar um troço qualquer pros meninos darem pra mãe deles domingo" (EEMC, p. 54) ou agaçante: "vontade de mandar tudo à, a mensalidade do curso de informática, as prestação do aparelho-de-dentes, o presentinho para o Dia das Mães, o cedê prometido à irmã caçula" (EEMC, p. 51). Pour le médecin "De branco" de garde de nuit appelé tantôt par sa femme, tantôt par une probable maîtresse, la fête des mères apparaît juxtaposée à plusieurs mots inaudibles de sa femmes à cause de la mauvaise qualité du réseau: "Amanhã (inaudível) pra comprar (inaudível) das mães" (EEMC, p. 115). La fête peut prendre plus d'ampleur dans d'autres fragments: elle explique le long déplacement en car accompli par la mère du sixième fragment et a probablement motivé la "Lettre" par laquelle la mère du fragment 50 tente se rapprocher du fils Paulino, qui, prénom oblige, vit maintenant à São Paulo. Mais personne ne prend la fête des mères plus au sérieux que le jeune délinquant - honteux et sans beaucoup d'expérience surnommé Brabeza. Si pour ceux qui peuvent avoir accès à la consommation, le cadeau reste une tâche presque bureaucratique à accomplir, pour Brabeza, qui n'a "pas un foutu rond dans la poche", l'achat du cadeau devient un véritable enjeu. Faire de menus larcins apparaît comme la seule solution envisageable pour se procurer le radiocassette qu'il veut offrir à sa mère malade: "a velha, coitada, nem exigente, aliás, nem esperando nada, o que ganhasse surpresa, de bom tamanho, aplaudiria" (EEMC, p. 44). Outre de faire plaisir à sa mère, l'idée de payer comptant le "gros cadeau" semble promettre à Brabeza une place, bien que provisoire, dans la société.

Par ailleurs, le match entre le Corinthians et le Rosário Central comptant pour la coupe Libertadores da América, qui a effectivement eu lieu ce 9 mai 2000, contribue à inscrire le texte de Ruffato dans la réalité, mais aussi dans la temporalité. Ce match est mentionné par plusieurs personnages, qui l'attendent tout au long de la journée: "Tem Corinthians hoje... Num vai não?" (EEMC, p. 50); "quanto você acha que vai ficar o jogo do corinthians está apostado uma cerveja" (EEMC, p.54). On apprend le résultat par les bruits de la rue, presque à la fin du livre, dans le fragment "67. Insônia": "alguém passa gritando corinthians, corinthians, coringão, ê, ê, ê, fogos de artifício" (EEMC, 152). Ruffato va jusqu'à placer le fragment "56. Slow Motion" dans le stade du Pacaembu au moment du match. Là où les caméras de télévision ne pénètrent pas, Marlon reconnaît le braqueur de son atelier de réparation de voitures placé juste derrière lui dans les 
gradins. La violence des émotions de ces deux supporters ennemis se confondent alors avec celles du match.

Si l'analyse que nous venons de présenter de la fragmentation et de ses connecteurs rend évidente la présence d'une critique sociale qui se "mimétise" à son objet, le choix des perspectives narratives nous permettra, désormais, de mieux comprendre l'éthique de Luiz Ruffato.

\section{L'engagement postmoderne}

La multiplication des instances narratives dans Eles eram muitos cavalos révèle différentes perspectives de la ville. Chaque fragment annonçant un nouveau narrateur, le lecteur doit identifier 69 fois le lieu d'où on parle et d'où on regarde, se forçant par là à se mettre à la place d'un autre, dans un exercice de décentrement permanent. Les diverses solutions narratives explorées par Ruffato vont des textes qui se présentent comme ce que j'ai appelé auparavant les "résidus textuels de la ville", où la focalisation au degré zéro efface le narrateur, jusqu'aux récits homodiégétiques et autodiégétiques. Cependant, la majorité des fragments contiennent des récits hétérodiégétiques, préférence qui ressort dès le titre du roman en portugais: Eles eram muitos cavalos, littéralement Ils étaient tant de chevaux.

D'une part, le narrateur à la troisième personne, chez Ruffato, n'a rien d'un intermédiaire autoritaire qui filtre la pensée et la parole de ses personnages. Bien au contraire, le recours au discours indirect libre, sans compter la place laissée aux monologues intérieurs et au flux de conscience des personnages, permet, comme nous l'avons vu, de donner la parole aux exclus. D'autre part, le choix de la troisième personne offre un mouvement de distance et de répulsion qui, selon l'écrivain et essayiste brésilien Silviano Santiago ${ }^{17}$, est le propre du narrateur postmoderne, qu'il définit comme: "celui qui veut s'extraire de l'action narrée, dans une attitude proche de celle du reporter ou d'un spectateur" (SANTIAGO, 2002, p.51), donc, "l'observateur d'un vécu qui lui est étranger" (SANTIAGO, 2002, p.46). Pour Santiago, la mise à distance permet à la fiction postmoderne d'évoquer "l'incommunicabilité de l'expérience" qui, nous l'avons vu, est un symptôme d'une crise de la représentation. Ce que le narrateur postmoderne communique est finalement l'expérience du regard. Santiago l'explique à travers cette belle image: “Un pont fait de mots enveloppe l'expérience muette du regard et rend possible le récit".

\footnotetext{
${ }^{17}$ Dans “O narrador pós-moderno", Santiago part de l'étude sur l'évolution des narrateurs entreprise par Walter Benjamin dans "Le Conteur: Réflexions sur l'œuvre de Nicolas Leskov". Il bâtit une typologie du narrateur postmoderne à partir d'une analyse de nouvelles de l'écrivain brésilien Edilberto Coutinho (SANTIAGO, 2002).
}

Ainsi, chez Ruffato, le choix du recyclage textuel, du collage et de la juxtaposition des voix et des discours, allant, par exemple, jusqu'à mettre l'homme et l'ordure au même niveau, communique l'indicible des scènes narrées. Cependant, contrairement à la majorité des écrivains et producteurs culturels contemporains, Ruffato n'utilise pas la pauvreté et la dégradation pour provoquer le dégoût ou un effet esthétique, tombant par-là dans ce que lui-même appelle néonaturalisme (voir note 8). La spécificité des narrateurs postmodernes de Eles eram muitos cavalos est justement le regard solidaire avec les personnages. Regard qui, malgré la distance imposée par la troisième personne, provoque chez le lecteur de la compassion pour les victimes et un malaise par rapport à l'injustice sociale.

Nombreuses sont les solutions narratives qui permettent à Ruffato de concilier distance et empathie, ou ce que Maria Zilda Cury appelle le "regard-avec les personnages" (CURY, 2007, p. 112). Souvent, l'empathie passe par l'adoption des points de vue des personnages, allant jusqu'à montrer ce qu'ils voient. Dans le fragment "6. Mãe", le paysage vu du car devient une longue énumération: "as cercas de arame farpado, as achas, o capim [...] a caatinga, os campos, a cana, a corda, corgo, o rio, o riacho, o riinho, o fio d'água [...]" (EEMC, p. 18-19). De même, dans le fragment "10. O que quer uma mulher", les regards des personnages deviennent révélateurs de leurs ambitions dans la vie: le mari, astigmate, apparaît "ligeiramente inclinado para proporcionar foco" (EEMC, p. 25), tandis que la femme, en ouvrant la fenêtre, permet au lecteur de voir avec elle l'extérieur et d'écouter les bruits de la rue. Ruffato parie aussi sur des perspectives inusitées, comme celle du corniaud du fragment " 11 . Chacina $\mathrm{n}$ 41 ” qui cherche son maître parmi les corps d'autres SDF assassinés. Une radicalisation de cette perspective a lieu lorsque le narrateur omniscient a accès au conscient et à l'inconscient des personnages ou adopte leur langage. C'est notamment le cas du fragment "67. Insônia" où les pensées, les souvenirs, les rêves et les bruits de la rue se mélangent. Le narrateur démiurge peut s'introduire dans une maison et mettre à nu l'intimité d'un foyer en énumérant les objets rangés dans l'office (fragment 32). Il va jusqu'à tomber par terre avec l'évangéliste (fragment 27) qui fait un malaise pendant sa prédication enflammée au centre-ville. Avec l'évangéliste, il regarde alors la ville depuis le sol, jusqu'à ce que le personnage puisse se relever: "alguns segundos? minutos? um par de sapatos um par de tênis solas gastas aproximam-se bitucas folhas copos descartáveis pombos guardanapos palitos papéis de bala poça de mijo 'Tudo bem aí ?' 'Tudo... Tudo bem..."” (EEMC, p. 63).

Une fois compris que, en ce qui concerne les fragments narratifs, l'éthique des divers narrateurs se fait sentir par 
cette distance solidaire, il nous reste à discuter l'enjeu de l'auteur. Au niveau global, aucune voix ne contrôle l'ensemble des fragments, ce qui crée une impression de morceaux, de scènes et de personnages de São Paulo juxtaposés au hasard formant une sorte d'échantillon représentatif de cette ville lacérée. Or, à défaut d'être un roman, cet échantillon recueilli dans une journée à São Paulo constitue bel et bien une œuvre littéraire et l'impression de hasard n'existe que parce que l'auteur le veut ainsi. La forme fragmentaire, tout en donnant l'impression d'effacer l'instance créatrice, l'expose d'avantage par l'absence d'un narrateur unique au niveau global. Mais si l'auteur endosse le rôle du chiffonnier qui recueille dans les déchets des rues des impressions papier des chaînes de prière, du collectionneur qui découpe avec soin les petites annonces et de l'observateur attentif aux scènes des rues et des foyers, il est surtout celui qui parodie ces textes. En outre, à l'instar d'un reporter, il monte son matériau, juxtapose des bouts de textes et, en le faisant, il se place ouvertement du côté des exclus.

Considérant comme Hutcheon (1991, p.21) que le roman postmoderniste est par définition autoréflexif, nous pouvons nous demander si Luiz Ruffato figure dans le texte à travers des narrateurs construits à son image. Les deux narrateurs-scripteurs du livre - la mère expéditrice de la lettre du fragment 50, avec ses phrases toutes faites et son accent provincial, et Paulo Sérgio Módena, le narrateur-scripteur du fragment "63. Nosso encontro" ne se posent à aucun moment des questions de fond et de forme sur l'acte d'écrire et ne mettent pas en abyme l'acte scriptural de Ruffato, car ils produisent un fragment précis et n'explicitent pas de lien avec les autres fragments du roman. Dans les quinze fragments avec un narrateur à la première personne, ce dernier peut être plus ou moins présent dans l'intrigue. Le narrateur occupe d'emblée une place centrale dans la diégèse dans: "20. Poderíamos ter sido grandes amigos", "41. Táxi" "46. O prefeito não gosta que lhe olhem nos olhos", "47. O "Crânio", "51. Política", "55. Via Internet", "58. Malabares", "61. Noite", "62. Da última vez", "63. Nosso encontro" et "64. Engradados". Dans les fragments "14. Índio", "33. A vida antes da morte" et "45. Vista parcial da cidade", les narrateurs occupent une place discrète laissant échapper l'emploi de la première personne à la fin du fragment.

La question de l'engagement apparaît dans le fragment "61. Noite", où le narrateur, abordé par une fille qui vend des bonbons, lui offre un repas dans une chaîne de fast-food libanais. Conscient que son action est provisoire, Humberto s'exclame: “jamais ne va me quitter ce malaise, jamais non plus cette sensation d'inutilité." Il est le seul narrateur qui pose directement le problème de l'engagement personnel dans la recherche d'une solution pour l'exclusion sociale au Brésil.

Le malaise d'Humberto est celui de Ruffato et probablement celui du lecteur. Mais quelle attitude adopter face à la violence, à l'inégalité et à la corruption ? Justement, dans le dernier fragment du livre, le seul à ne pas avoir de titre, un couple se demande comment agir face à une situation de violence. En pleine nuit, ils entendent les gémissements de quelqu'un qui a probablement été poignardé devant leur porte: “- Não vamos ajudar?" demande la femme. "- Ficou doida?", est la réponse du mari. "- Por quê?", interroge encore la femme. La sensation répandue au Brésil de pouvoir être la prochaine victime motive la réponse qui achève la conversation et le livre : "- Porque... porque ainda pode ter alguém lá... E aí? Melhor dormir... Vai... vira pro canto... vira pro canto e dorme... Amanhã... amanhã a gente vê... Amanhã a gente fica sabendo... Dorme... Vai" (EEMC, p. 158). Le lecteur, lui, pourra-t-il se retourner et s'endormir à la fin du livre?

Le fragment "63. Notre rendez-vous", à son tour, évoque, très implicitement, le cheminement de l'engagement politique des années 1980 jusqu'à présent. Explicitement, le narrateur, écrivant à dix heures et demie du soir dans un bar, après avoir consommé neuf bières, parle de ses amis quarantenaires, attablés avec lui, de leurs soucis et des siens avec "amour et sordidité". De plus, Paulo Sérgio Módena affirme dessiner un tableau "[d]as idas e vindas da classe média brasileira nos últimos quinze anos" (EEMC, p. 136). Le groupe est formé "por gente de vária procedência, intelectual e social" (EEMC, p. 136), tous issus des mouvements politiques étudiants, qui, à la fin du régime militaire brésilien, réclamaient l'amnistie des prisonniers politiques. Le début des rendez-vous annuels coïncide significativement avec le mouvement "Diretas já" (1984), qui réclamait des élections démocratiques immédiatement après la fin du dernier gouvernement militaire. Que sont donc devenus leurs idéaux aujourd'hui ? À part Pierre, médecin raté et encore fervent communiste, l'engagement politique a complètement disparu du groupe. Ils se réunissent maintenant par simple habitude, par solitude, ou peutêtre parce que la rencontre du 9 mai fonctionne comme un repère. Ce récit, à travers l'histoire individuelle de chaque membre du groupe, raconte, finalement, celle de la disparition des derniers grands idéaux au Brésil après la fin de la dictature.

Luiz Ruffato, tout en se montrant conscient de ce vide idéologique, propose à son lecteur de ne pas fermer les yeux sur l'inégalité. En effet, comme nous l'avons vu, Eles eram muitos cavalos offre plusieurs points de vue de São Paulo; significativement, les regards des individus de cette mégalopole éclatée ne se croisent jamais, tout 
comme les personnages ne réapparaissent pas d'un texte à l'autre. Ainsi, dans un livre qui se constitue comme un zapping soigneusement agencé, aucune solution satisfaisante n'apparaît; à chaque fragment, seulement de nouvelles questions sur lesquelles réfléchir. Et c'est justement là que, paradoxalement, se trouve l'engagement de Luiz Ruffato dans Eles eram muitos cavalos.

\section{Referências}

BAUMAN, Zygmunt. Vie perdues: la modernité et ses exclus. Traduit de l'anglais par Monique Bégot. Paris: Payot \& Rivages, 2009.

BOSI, Alfredo. Situações e forma do conto brasileiro contemporâneo. In: São Paulo: Cultrix, 2002. (Org.). O Conto brasileiro contemporâneo.

BUARQUE DE HOLLANDA, Heloísa. Entrevista com luiz Ruffato. Disponible sur: $<$ http://www.heloisabuarquedehollanda. com.br/?p=706>. Consultado em: 31 out. 2016.

CANDIDO, Antonio. Dialética da Malandragem caracterização das Memórias de um sargento de milícias. Revista do Instituto de Estudos Brasileiros, São Paulo, USP, n. 8, p. 67-89, 1970.

CANDIDO, Antonio. L'endroit et l'envers: essai de littérature et de sociologie. Paris: Métailié-Unesco, 1995.

CORPAS, Danielle. De boas intenções o inferno está cheio. Cerrados: Revista do Programa de Pós-graduação em Literatura, Brasília, UNB, ano 18, n. 28, 2009.

CURY, Maria Zilda. Ética e simpatia: o olhar do narrador em contos de Luiz Ruffato. In: HARRISON, Marguerite Itamar (Org.). Uma cidade em camadas: ensaios sobre Eles eram muitos cavalos de Luiz Ruffato. Vinhedo: Horizonte, 2007.

EAGLETON, Terry. As ilusões do pós-modernismo. Rio de Janeiro: Zahar, 1998.
HARRINSON, Marguerite Itamar (Org.). Uma Cidade em camadas: ensaios sobre Eles eram muitos cavalos de Luiz Ruffato. Vinhedo: Horizonte, 2007.

HUTCHEON, Linda. Poética do pós-modernismo: história, teoria, ficção. Traduit par Ricardo Cruz. Rio de Janeiro: Imago, 1991.

JAMESON, Frederic. Pós-modernismo: a lógica do capitalismo tardio. São Paulo: Ática, 2004.

MEIRELLES, Cecília. Romanceiro da Inconfidência. In: Poesia Completa. Rio de Janeiro: Nova Fronteira, 2001. Vol. 1.

ROCHA, João Cézar Castro. La dialectique de la 'marginalité' et la violence dans la culture brésilienne contemporaine. Littérature du Brésil, Europe, n. 919-920, p. 70-71, nov.-déc., 2005.

RUFFATO, Luiz. Tant et tant de chevaux. Traduit du brésilien par Jacques Thiériot. Paris: Métailié, 2005.

RUFFATO, Luiz. Eles eram muitos cavalos. 7. ed. rev. Rio de Janeiro: Record, 2007.

SANTIAGO, Silviano. O narrador pós-moderno. In: Nas malhas da letra. Rio de Janeiro: Record, 2002.

SCHØLLHAMMER, Karl Erik. Fragmentos do real e o real dos fragmentos. In: HARRISON, Marguerite Itamar (Org.). Uma Cidade em camadas: ensaios sobre Eles eram muitos cavalos de Luiz Ruffato. Vinhedo: Horizonte, 2007.

SCHØLLHAMMER, Karl Erik. Ficção brasileira contemporânea, Rio de Janeiro: Civilização Brasileira, 2009.

WALTY, Ivete Lara Camargos. Anonimato e resistência em Eles eram muitos cavalos. In: HARRISON, Marguerite Itamar (Org.). Uma Cidade em camadas: ensaios sobre Eles eram muitos cavalos de Luiz Ruffato. Vinhedo: Horizonte, 2007.

Recebido: 01 de junho de 2016.

Aprovado: 15 de junho de 2016.

Contato: Ilana.Heineberg@u-bordeaux-montaigne.fr 